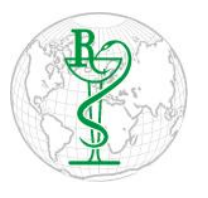

INDO GLOBAL JOURNAL OF

PHARMACEUTICAL SCIENCES

ISSN 2249- 1023

\title{
An Overview on Traditional Aphrodisiac Plants found in Maharashtra, India
}

\author{
Ghumare J. G., N.R. Somwanshi, B. M. Gaykar \\ Department of Botany, Ahmednagar College, Ahmednagar (MS) \\ Address for Correspondence: N.R. Somwanshi, nr.somvanshi@ rediffmail.com
}

\begin{abstract}
Received:
01.02.2019

Accepted:

20.08.2019

Keywords

Aphrodisiac,

Medicinal Plants,

Traditional

Medicines.
\end{abstract}

ABSTRACT: An agent either food or drug that arouses sexual desire is called "aphrodisiac". The word is derived from Aphrodite, i.e. the Greek goddess of sexual, love \& beauty. Since ancient time, man uses methods to increase his sexual power either by ritual dances or hunting. History reveals importance of plants as aphrodisiac agents. Sexual relation is important in social \& biological relationship in human life. Erectile dysfunction is persistent inability to obtain \& maintain erection for naturally satisfying intercourse, it is a condition that affects millions of people worldwide. (C) 2019 iGlobal Research and Publishing Foundation. All rights reserved.

Cite this article as: Ghumare, J.G.; Somwanshi, N.R.; Gaykar, B.M. An overview on traditional aphrodisiac plants found in Maharashtra, India. Indo Global J. Pharm. Sci., 2019; 9(2): 91-97. DOI: http://doi.org/10.35652/IGJPS.2019.9208.

\section{INTRODUCTION}

An agent either food or drug that arouses sexual desire is called "aphrodisiac". The word is derived from Aphrodite, i.e. the Greek goddess of sexual, love \& beauty. Since ancient time, man uses methods to increase his sexual power either by ritual dances or hunting. History reveals importance of plants as aphrodisiac agents. Some substances used as aphrodisiac includes Yohimbine \& Mandrake plants in Africa, Europe \& ground Rhinoceros horn in China (Klinger et al., 1996). Sexual relation is important in social \& biological relationship in human life. Erectile dysfunction is persistent inability to obtain \& maintain erection for naturally satisfying intercourse, it is a condition that affects millions of people worldwide.

Sexual dysfunction is a serious medical \& social problem observed in an average of $32 \%$ of males. A population based study in US revealed that prevalence of MSD was $12 \%$ in those younger than 59 years, $22 \%$ in those $60-69$ years of age, \& 30\% in those older than 69 years. (Bacon et al., 2003). As per an estimate over 320 million people in the Westernized nations will be develop MSD by 2025 (McKinlay JB, 2000).
It is a factor contributing to infertility (Yakubu et al., 2003). The problem occurs mainly in middle aged \& older men affected by diabetes, hypertension, depression \& due to drugs (Guay et al., 2003)

The method of treatment for erectile dysfunction involves several aphrodisiac methods such as vacuum erection devices, surgical Penile Implants, Hormonal treatments (Testosterone) or use of specific drugs like Sildenafil (Viagra), Tadalafil (Cialis), Vardenafil (Levitra) etc. These treatments have some serious side effects, or complications like infections, failure of devices, acceptability, headache, flushing, dizziness, visual disturbances, congestion, priapism, hypertension etc. The treatments are also very costly.

Medicinal plants are used since ancient times; plants can be used directly or in powdered or extract form. Due to their various phytochemical properties, they are used for management of various diseases. 
Indo Global Journal of Pharmaceutical Sciences, 2019; 9(2): 91-97

Medicinal plants are used as natural supplements because of their fewer side effects, ready availability \& less cost. Plants show significant pharmacological activity. Herbs can be effectively aphrodisiac, moreover isolation $\&$ identification of active constituents from plants may bring a dynamic change in the modern world (Malviya et al., 2011)

Best uses of aphrodisiac with no or less side effects are of plant origin. First known such aphrodisiac is Yohimbine from the bark of Yohimbe tree used in West Africa. (Klinger et al., 1996). Other known aphrodisiac includes, Ginseng, Ginger, Ginkgo, Eurycoma etc. African people use Aloe, Pumpkin etc since years to stimulate \& improve sexual performance (Kamtchouing et al., 2002; Orisakwe et al., 2004).

Experimental studies are performed to define claim of efficacy. Most studies targeted one plant at a time (Ratnasooriya \& Dharmasiri, 2000). But traditionally two or more plants are used in combination.

For the determination of the safety \& effectiveness of these substances for sexual enhancement, it is necessary to test preclinically in animals \& clinically in human being before consuming these drugs. So, demands of natural aphrodisiac require increasing studies to understand their effects on humans. (Patel, 2011)

The traditional aphrodisiac plants contain active ingredients that includes terpenoids, alkaloids, cardiac glycosides, saponins \& flavonoids ( Birdis et al., 2008).

The chief mechanism of aphrodisiac action shown by a number of medicinal plants is increase in Serum Testosterone level. Ethanolic extract of Blepharis edulis Forssk. roots (Chatterjee et al., 1990), aqueous extracts of Massularia acuminate (G. Don) Bullock ex Hoyle roots (Yakubu et al., 2008), exhibits aphrodisiac activity by enhancing testosterone level. Even they enhance FSH \& LH levels. Panax ginseng C.A.Mey. show aphrodisiac activity by nitric oxide linked mechanisms; reports showed that it enhances Nitric Oxide synthesis resulting in relaxation of Corpus Carenosum in penis \& increase in penile rigidity \& girth. (Dubey et al., 1997 \& Jirovetz et al., 2005). Further studies are also needed to check the mechanism behind the activity in traditional aphrodisiac plants in combination.

Literature study reveals that few research papers are available on plant improving fertility or impotency in men ( Evans,1969; Pallavi et al, 2011; Neychev, 2005, Sabna et al, 2013)

State of Maharashtra is rich in biodiversity. Many plants are used as medicinal and for variety of ayurvedic preparation for curing diseases and related problems since ancient times. Present study enlists the plants with aphrodisiac properties specifically from Maharashtra, India.

\section{METHODOLOGY}

Survey of Aphrodisiac plants found all over the world was carried by many people, including Ramandeep Singh, et al, 2012. The plants enlisted by Ramandeep Singh and coworkers were taken into consideration and plants found in flora of Maharashtra were sorted out.

\section{RESULTS \& DISCUSSION}

The present review was carried out pertaining to the aphrodisiac plants. The results are very interesting that the plants reported in one location, also cross checked for another region. The use of plant or plant parts as aphrodisiac was found same.

Table: Review of Aphrodisiac plants in Maharashtra, India.

\begin{tabular}{|c|c|c|c|c|}
\hline $\begin{array}{c}\text { Sr. } \\
\text { No. }\end{array}$ & Name of plant & Family & Part used & Reference \\
\hline 1 & Abelmoschus esculentus (L.) Moench & Malvaceae & Root & Khan \& Khan, 2005 \\
\hline 2 & Abelmoschus moschatus Medik & Malvaceae & Seed & $\begin{array}{c}\text { Joy PP, et al. 2000 \& } \\
\text { Meena KA, et al, 2009 }\end{array}$ \\
\hline 3 & Abrus precatorius L. & Fabaceae & seed & Meena KA, et al, 2009 \\
\hline 4 & Abutilon indicum (Linn) Sweet. & Malvaceae & $\begin{array}{c}\text { seed, root, bark, } \\
\text { leaf }\end{array}$ & Joy PP, et al. 2000 \\
\hline 5 & Acacia catechu Willd. & Mimosaceae & Heartwood & Joy PP, et al 2000 \\
\hline 6 & Acacia niloltica (L.) Willd. ex Delile & Fabaceae & Bark & Chandra Prakash Kala, 2005 \\
\hline 7 & Achyranthes aspera L. & Amaranthaceae & Root & Khan \& Khan, 2005 \\
\hline
\end{tabular}


Indo Global Journal of Pharmaceutical Sciences, 2019; 9(2): 91-97

\begin{tabular}{|c|c|c|c|c|}
\hline 8 & Acorus calamus L. & Acoraceae & Rhizome & $\begin{array}{l}\text { Kapoor LD, } 1990 \text { and } \\
\text { Willium JJ, et al, } 1970\end{array}$ \\
\hline 9 & Adenanthera pavonina L. & Mimosaceae & Bark,Seeds,leaves & Agarwal SS, 2005 \\
\hline 10 & Allium sativum $\mathrm{L}$. & Amaryllidaceae & Bulb & $\begin{array}{c}\text { Joy PP, et al 2000; Meena KA, et al, 2009; } \\
\text { Chopra, } 2002\end{array}$ \\
\hline 11 & Allium cepa $\mathrm{L}$. & Amaryllidaceae & Bulb & Malviya N, et al., 2011 \\
\hline 12 & Aloe vera (L.) Burm.f & Asphodelaceae & $\begin{array}{c}\text { Gel extracted from } \\
\text { leaves }\end{array}$ & Malviya N , et al., 2011 \\
\hline 13 & Alpinia galanga (L.) Willd. & Zingiberaceae & Rhizome & Anand RM, et al, 2006 \\
\hline 14 & Amaranthus spinosus L. & Amaranthaceae & Whole plants & Khan \& Khan, 2005 \\
\hline 15 & Asparagus reacemosus Willd. & Asparagaceae & Root & $\begin{array}{c}\text { Satyavati GV, et al, } 1976 \text { \& Dange PS, et } \\
\text { al, } 1969\end{array}$ \\
\hline 16 & Arachis hypogaea L. & Fabaceae & Seed & Agarwal SS, 2005 \\
\hline 17 & Argyreia nervosa (Burm f.) Bojer & Convolvulaceae & Root & Subramoniam A, et al, 2007 \\
\hline 18 & Artocarpus heterophyllus Lam. & Moraceae & $\begin{array}{c}\text { Fruit, Seed, Leaves, } \\
\text { root }\end{array}$ & Agarwal SS, 2005 \\
\hline 19 & Aristolochia indica L. & Aristolochiaceae & Whole plant & Malviya N, et al., 2011 \\
\hline 20 & Atylosia scarabaeoides (L.) Benth. & Fabaceae & Whole plant & Malviya N, et al., 2012 \\
\hline 21 & Azadirachta indica $\mathrm{L}$. & Meliaceae & Root & Khan \& Khan, 2005 \\
\hline 22 & Bacopa monnieri (L.) Pennell & Plantaginaceae & Whole plant & Khan \& Khan, 2005 \\
\hline 23 & Bauhinia tomentosa $\mathrm{L}$. & Caesalpinaceae & Seed & Joy PP, et al, 2000 \\
\hline 24 & Bauhinia vahlii Wight and Arn. & Caesalpinaceae & Seed & Joy PP, et al, 2000 \\
\hline 25 & Bauhinia variegata (L.) Benth. & Caesalpinaceae & Bark & Joy PP, et al, 2000 \\
\hline 26 & Benincasa hispida (Thunb.) Cogn. & Cucurbitaceae & Fruit & Joy PP, et al, 2001 \\
\hline 27 & Boerhavia diffusa $\mathrm{L}$. & Nyctaginaceae & Root & Khan \& Khan, 2005 \\
\hline 28 & Bombax ceiba L. & Bombacaceae & Bark & Joy PP, et al, 2001 \\
\hline 29 & Boesenbergia rotunda (L.) Mansf. & Zingiberaceae & Rhizome & $\begin{array}{c}\text { Sumalatha K, et al., } 2010 \text { \& Ching LYA, et } \\
\text { al., } 2007\end{array}$ \\
\hline 30 & Bryonia laciniosa Linn. & Cucurbitaceae & Seed & Malviya N, et al., 2012 \\
\hline 31 & Butea frondosa Roxb. & Fabaceae & Whole plant & $\begin{array}{c}\text { Joy PP, et al, } 2001 \& \\
\text { Sumalatha K, et al., } 2010\end{array}$ \\
\hline 32 & Cajanus cajan (L.) Millsp. & Fabaceae & Root & Khan \& Khan, 2005 \\
\hline 33 & Carica papaya $\mathrm{L}$. & Caricaceae & Fruit & Khan \& Khan, 2005 \\
\hline 34 & Cannabis sativa $\mathrm{L}$. & Cannabinaceae & Leaf & Khan \& Khan, 2005 \\
\hline 35 & Capsicum annuиm $\mathrm{L}$. & Solanaceae & Root & Elferink RGJ, 2000 \\
\hline 36 & Cassia occidentalis Linn & Fabaceae & Leaf & Singh KP, et al., 2010 \\
\hline 37 & Cassia sieberianan DC & Caesalpinaceae & Leaf & Sugiyama Y, et al., 1992 \\
\hline 38 & Celastrus paniculatus Willd. & Celastracae & Seeds & Malviya N, et al., 2012 \\
\hline 39 & Chenopodium album L. & Chenopodiaceae & Seed & $\begin{array}{l}\text { Jaiswal S, et al., } 2004 \& \\
\text { Vanwyk BE, et al., } 2000\end{array}$ \\
\hline 40 & Chlorophytum tuberosum Baker. & Liliaceae & Whole plant & Maiti S, et al., 2007 \\
\hline 41 & Chlorophyllum arundinaceum Baker. & Liliaceae & Root & Malviya N , et al., 2012 \\
\hline 42 & Cissus quadrigularis Linn. & Vitaceae & Root & Joy PP, et al, 2001 \\
\hline 43 & Cocculus cordifolius Linn. & Menispermaceae & Stem, Leaf, Root & Prasanth PR, Kumar A, 2008 \\
\hline 44 & Cocus nucifera Linn & Arecaceae & Endosperm & $\begin{array}{c}\text { Meena KA, et al, } 2009 \& \\
\text { Dahanukar SA, } 1989\end{array}$ \\
\hline
\end{tabular}


Indo Global Journal of Pharmaceutical Sciences, 2019; 9(2): 91-97

\begin{tabular}{|c|c|c|c|c|}
\hline 45 & Commiphora mukul Hook. Ex Stocks & Burseraceae & root, leaf & Joy PP, et al, 2001 \\
\hline 46 & Coriandrum sativum Linn. & Apiaceae & Leaf & Jaiswal S, et al., 2004 \\
\hline 47 & Curcuma amada Roxb. & Zingiberaceae & Rhizome & $\begin{array}{l}\text { Joy PP, et al, } 2001 \& \\
\text { Thomas, et al., } 2000\end{array}$ \\
\hline 48 & Curcuma anguistifolia Roxb. & Zingiberaceae & Rhizome & Malviya N, et al., 2012 \\
\hline 49 & Curcuma aromatica Roxb. & Zingiberaceae & Rhizome & Malviya N, et al., 2012 \\
\hline 50 & Cymbopogon citratus (DC.) & Poaceae & whole plant & Joy PP, et al, 2001 \\
\hline 51 & Dalbergia sissoo Roxb. & Fabaceae & wood & $\begin{array}{c}\text { Singh KP, } 2010 \& \\
\text { Aiswal S, et al., } 2004\end{array}$ \\
\hline 52 & Daucus carota $\mathrm{L}$. & Apiaceae & Root & Woys WW., 1997 \\
\hline 53 & Desmodium gangeticum Linn & Fabaceae & Root & Joy PP, et al, 2001 \\
\hline 54 & Dioscorea bulbifera Linn. & Dioscoreaceae & Whole plant & Singh KP, 2010 \\
\hline 55 & Diospyros melanoxylon Roxb. & Ebenaceae & Flower & $\begin{array}{c}\text { Joy PP, et al., } 2001 \& \\
\text { Singh KP, } 2010\end{array}$ \\
\hline 56 & Dolichos lablab Linn. & Fabaceae & Seeds & Agrawal SS., 2005 \\
\hline 57 & Drypetes roxburghii (Wall) Huru. & Euphorbiaceae & Leaf juice & Singh KP, 2010 \\
\hline 58 & Emblica officinalis Gaertn. & Euphorbiaceae & Fruit & $\begin{array}{c}\text { Cythia W., } 1993 \& \\
\text { Ahmad SS., } 2007\end{array}$ \\
\hline 59 & Euphorbia hirta L. & Euphorbiaceae & Leaves & Sugiyama Y, et al., 1992 \\
\hline 60 & Evolvulus alsinoides L. & Convolvulaceae & Whole plant & Agrawal SS., 2005 \\
\hline 61 & Ficus arnottiana Miq. & Moraceae & Bark & Bakshi DNG, et al., 2001 \\
\hline 62 & Ficus racemosa L. & Moraceae & Fruit & Khan \& Khan, 2005 \\
\hline 63 & Ficus religiosa Linn. & Moraceae & Bark & Joy PP, et al, 2001 \\
\hline 64 & Ficus retusa $\mathrm{L}$. & Moraceae & Latex & Swapnadeep Parial, et al., 2010 \\
\hline 65 & Ficus bengalensis L. & Moraceae & Latex & Husain A, et al., 1992 \\
\hline 66 & Gossypium arboreum Linn. & Malvaceae & $\begin{array}{l}\text { Bark, Seeds, } \\
\text { Leaves, Fruit }\end{array}$ & Agrawal SS., 2005 \\
\hline 67 & Grewia asiatica $\mathrm{L}$. & Tiliaceae & Fruit & Aiswal S, et al., 2004 \\
\hline 68 & Hibiscus rosa-sinensis & Malvaceae & Leaves & Joy PP, et al, 2001 \\
\hline 69 & Hibiscus lobatus Murr. & Malvaceae & Whole plant & Malviya N, et al., 2012 \\
\hline 70 & Hibiscus sabdariffa Linn. & Malvaceae & Seed, leaf & Joy PP, et al, 2001 \\
\hline 71 & Holostemma ada-kodien Schult. & Asclepiadaceae & Root & Joy PP, et al, 2002 \\
\hline 72 & Hygrophilia auriculata Schum. & Acanthaceae & Seeds & Malviya N, et al., 2012 \\
\hline 73 & Hygrophilia schulli (Ham.) & Acanthaceae & Root, leaf, Seeds & Joy PP, et al, 2001 \\
\hline 74 & Ipomoea mauritiana Jacq. & Convolvulaceae & Roots & Joy PP, et al, 2001 \\
\hline 75 & Jatropha curcas L. & Euphorbiaceae & Seeds & Malviya N, et al., 2012 \\
\hline 76 & Lagenaria vulgaris Ser. & Cucurbitaceae & Fruit & Joy PP, et al, 2001 \\
\hline 77 & Linum usitatissimum $\mathrm{L}$ & Linaceae & Seed & Khan \& Khan, 2005 \\
\hline 78 & Litsea chinensis Heyne ex. Nees & Lauraceae & Bark & Zamble A, et al., 2008 \\
\hline 79 & Mallotus philippensis Lam. & Euphorbiaceae & $\begin{array}{l}\text { Glandular hair on } \\
\text { Fruit }\end{array}$ & Malviya N, et al., 2012 \\
\hline 80 & Mangifera indica L. & Anacardiaceae & Bark & Joy PP, et al, 2001 \\
\hline 81 & Mimosa pudica L. & Mimosaceae & Aerial part & Sankaranarayanan S, et al., 2010 \\
\hline 82 & Mirabilis jalapa L. & Nyctaginaceae & Root & Ahmad SS, 2007 \\
\hline
\end{tabular}


Indo Global Journal of Pharmaceutical Sciences, 2019; 9(2): 91-97

\begin{tabular}{|c|c|c|c|c|}
\hline 83 & Momordica charantia L. & Cucurbitaceae & Leaf & Sharma VN, 1960 \\
\hline 84 & Mucuna pruriens (L.) DC. & Fabaceae & Seed & Singh KP, 2010 \\
\hline 85 & Murdannia edulis (Stokes) Faden & Commelinaceae & Dried root & Malviya N, et al., 2012 \\
\hline 86 & Myristica fragrans Houtt & Myristicaceae & Seed & $\begin{array}{c}\text { Joy PP, et al, } 2001 \& \\
\text { Sumalatha K, et al., } 2010\end{array}$ \\
\hline 87 & Nerium indicum Mill. & Apocynaceae & Root & Singh KP, 2010 \\
\hline 88 & Ocimum gratissimum $\mathrm{L}$. & Labiatae & Leaves & Joy PP, et al, 2001 \\
\hline 89 & Passiflora incarnate L. & Passifloraceae & Leaf & Gilman FE, 1999 \\
\hline 90 & Papaver somniferum L. & Papaveraceae & Flower & Joy PP, et al, 2001 \\
\hline 91 & Pedalium murex L. & Pedaliaceae & Whole Plant & Balamurugan $\mathrm{G}$, et al., 2010 \\
\hline 92 & Phyllanthus emblica L. & Phyllanthaceae & Fruit & Malviya N, et al., 2012 \\
\hline 93 & Piper betle L. & Piperaceae & Leaf & Sankaranarayanan S, et al., 2010 \\
\hline 94 & Prunus amygdalus Batsch & Rosaceae & Kernel & Agrawal SS., 2005 \\
\hline 95 & Psoralea corylifolia Linn. & Fabaceae & Fruit & Agrawal SS., 2005 \\
\hline 96 & Punica granatum $\mathrm{L}$. & Punicaceae & Fruit & Khan \& Khan, 2005 \\
\hline 97 & Ricinus communis L. & Euphorbiaceae & Seed & Joy PP, et al, 2001 \\
\hline 98 & Rosa damascene Mill & Rosaceae & Petal & Garg SC, 2005 \\
\hline 99 & Saccharum spontaneum Linn & Poaceae & Root stock & $\begin{array}{c}\text { Meena DA, et al., } 2009 \& \\
\text { Chopra RN, et al., } 2002\end{array}$ \\
\hline 100 & Santalum album L. & Santalaceae & Heartwood & Garg SC, 2005 \\
\hline 101 & Sesamum indicum $\mathrm{L}$. & Pedaliaceae & Seeds & Singh KP, 2010 \\
\hline 102 & Shorea robusta Roth. & Dipterocarpaceae & Bark, leaves, Fruit & Agrawal SS., 2005 \\
\hline 103 & Sida cordifolia $\mathrm{L}$. & Malvaceae & Root, Seed & Joy PP, et al, 2001 \\
\hline 104 & Sida acuta Burm.f. & Malvaceae & Whole Plant & Khan \& Khan, 2005 \\
\hline 105 & Sida rhombifolia $\mathrm{L}$. & Malvaceae & Root & Khan \& Khan, 2005 \\
\hline 106 & Solanum indicum $\mathrm{L}$. & Solanaceae & Root & Joy PP, et al, 2001 \\
\hline 107 & Solanum americanum Mill. Gard & Solanaceae & Whole Plant & Malviya N, et al., 2012 \\
\hline 108 & Solanum melongena L. & Solanaceae & Unriped Fruit & Joy PP, et al, 2001 \\
\hline 109 & Solanum nigrum Linn. & Solanaceae & Berries & Joy PP, et al, 2001 \\
\hline 110 & Sphaeranthus africanus L. & Asteraceae & Whole plant & Joy PP, et al, 2001 \\
\hline 111 & Sphaeranthus indicus L. & Asteraceae & Seeds & Khan \& Khan, 2005 \\
\hline 112 & Strychnos nux-vomica L. & Loganiaceae & Seed & Meena KA, et al., 2010 \\
\hline 113 & $\begin{array}{l}\text { Syzygium aromaticum (L.) Merrill \& } \\
\text { Perry }\end{array}$ & Myrtaceae & Dried flower bud & $\begin{array}{l}\text { Khan MA, } 1886 \& \\
\text { Baytar I, et al, } 1869\end{array}$ \\
\hline 114 & Tamarindus indica $\mathrm{L}$. & Fabaceae & Bark & Jain LD, et al., 2010 \\
\hline 115 & Tamarix aphylla (L.) Karst & Tamaricaceae & Bark & Jaiswal S, et al., 2004 \\
\hline 116 & Terminalia arjuna (Roxb.) Wight \& Arn & Combretaceae & Bark & Joy PP, et al, 2001 \\
\hline 117 & Tinospora cordifolia (Willd) Miers & Menispermaceae & Whole plant & $\begin{array}{l}\text { Joy PP, et al, } 2001 \& \\
\text { Thomas, et al., } 2000\end{array}$ \\
\hline 118 & Tribulus terrestris L. & Zygophyllaceae & Fruit, Seed & $\begin{array}{l}\text { Singh KP, } 2009 \& \\
\text { Neychev VK, } 2005\end{array}$ \\
\hline 119 & Tricholepis glaberrima DC & Asteraceae & Aerial part & Zamble A, et al., 2008 \\
\hline 120 & Trichosanthes dioica L. & Cucurbitaceae & Seed & Joy PP, et al, 2001 \\
\hline
\end{tabular}


Indo Global Journal of Pharmaceutical Sciences, 2019; 9(2): 91-97

\begin{tabular}{|c|c|c|c|c|}
\hline 121 & $\begin{array}{c}\text { Vanda tessellata (Roxb.) Hook. ex } \\
\text { G.Don. }\end{array}$ & Orchidaceae & Flower, Root & $\begin{array}{c}\text { Jain BJ, et al., 2006 \& } \\
\text { Suresh Kumar PK, et al., 2000 }\end{array}$ \\
\hline 122 & Withania somnifera (L.) Dunal & Solanaceae & Leaf, Root & $\begin{array}{c}\text { Joy PP, et al, 2001; Singh KP, 2010 \& } \\
\text { Aiswal S, et al., 2004 }\end{array}$ \\
\hline 123 & Wrightia tinctoria (Roxb.) R.Br. & Apocynaceae & Seed, leaf, bark & Joy PP, et al, 2001 \\
\hline 124 & Zingiber offininale Roscoe & Zingiberaceae & Rhizome & Mugisha MK, Origa HO, 2005 \\
\hline
\end{tabular}

\section{CONCLUSION}

Maharashtra state of India is rich in biodiversity even in terms of plants with aphrodisiac potentials. Out of 198 plant species reported earlier as aphrodisiac, Maharashtra contains 124 species of plants belongs to $\mathbf{1 0 2}$ genera of $\mathbf{5 8}$ Families. The plants enlisted in this reviews are natural and with aphrodisiac potentials. Some plants are used in ayurvedic formulation to overcome erectile dysfunction. Due to the side effects of allopathetic drugs, demand for plant based medication is increasing day by day. So the review helps scientists working on aphrodisiac plants, which gives details of scientific name, family, part used and reference.

\section{ACKNOWLEDGEMENTS}

Authors wish to express humble and sincere thanks to Honorable Principal, Dr. R.J. Barnabus, Ahmednagar College, Ahmednagar, for his intense support and providing necessary facilities to prepare this review.

\section{REFERENCES}

1. Agrawal SS. Clinically useful herbal drugs. Published by Ahuja Pub., Delhi, 2005, 100-123

2. Ahmad SS. Medicinal wild plants from Lahore- Islamabad motorway (m-2). Pak J Bot 2007; 39(2): 355-375

3. Anand RM, Nandakumar N, Karunakaran L, Ragunathan M, Murugan V. A Survey of medicinal plants in Kollimalai hill tracts, Tamil Nadu. Natural Products Radiance 2006; 5(2):139-143.

4. Bacon CG, Mittleman MA, Kawach I, Goivanncci E, Glassen DB, Rimm EB. Sexual function in men older than 50 years of age: Results from health Professionals follow up study. Annals of Internal medicine; 2003: 139(3) 161-168.

5. Bakshi DNG, Sharma PS, Pal DC. A Lexicon of Indian Medicinal Plants. Vol. 2, Naya prakashan, New Delhi, 2001, 190.

6. Balamurugan G, Muralidharan P, Polapala S. Aphrodisiac activity and curative effect of Pedalium murex (L.) against ethanol-induced infertility in male rats. Turk J Biololgy 2010; 34:153-163.

7. Baytar I, Kitabul Jame' Li-Mufradat il Advia walaghzia, Cairo, Egypt, Matba Zahiyah Zaaherah Mutawafferah, 1869; 5:7-9.

8. Birdi, T.J; Brijesh, S. \& Daswani, P.G. (2008): Approaches towards the preclinical testing \& standardization of medicinal plants. Foundations for Med. Research, India

9. Chandra Prakash Kala: Indigenous uses, population density and conservation of threatened medicinal plants in protected areas of the Indian Himalayas. Conservation Biology 2005; 19(2):368-378.

10. Chatterjee A, Sharma NJ, Bannerji, Basa SC. Studies on acanthaceaebenzoxazolone from Blepharis edulis Pers. Ind J of Chem. 1990; 29132134.

11. Chauhan N.S., Dixit V.K.: Int. J. Appl. Res. Nat. Prod. 1, 26, 2008.

12. Ching LYA, Wah ST, Sukari AM, Lian CEG, Rahmani M, Khalid K. Characterization of flavonoid derivatives from Boesenbergia rotunda (L.). The Malaysian Journal of Analytical Sciences 2007; 11(1):154-159.
13. Chopra RN, Nayar SL, Chopra IC. Glossary of Indian medicinal plants, NISCIR, CSIR, Delhi, 2002.

14. Cynthia W. Love Potions - a guide to aphrodisiacs. Optima Books, 1993.

15. Dahanukar SA, Thatte UM. Therapeutic approaches in Ayurveda Revisited. Poppular Prakashan, Mumbai, 1989, 109-110.

16. Dange PS, Kanitkar UK, Pendse GS. Amylase and lipase activities in the roots of A. racemosus Planta Medica 1969; 17:393.

17. Dubey NK, Kishore N, Varma J, Lee SY. Cytotoxicity of essential oils of Cymbopogon citratus \& Ocimum gratissimum. Indian J Pharm Sc. 1997; 59: 263-4.

18. Elferink RGJ. Aphrodisiac Use in Pre-Columbian Aztec and Inca Cultures. Journal of the History of Sexuality 2000; 9(1/2):25-36

19. Garg SC. Essential oils as Therapeutics. Natural Product Radiance 2005; 4(1):18-26.

20. Gilman FE. Passiflora incarnata -- Wild Passion Flower, Series of the Environmental Horticulture Department, Florida Cooperative Extension Service. Institute of Food and Agricultural Sciences, University of Florida, Fact Sheet FPS- 457, 1999, 1-3.

21. Guay AT, Spark RF, Bansal S, Cunningham GR, Goodman NF, Nankin $\mathrm{HR}$ et al., American association of Clinical Endocrinologist, Medical guidelines for Clinical Practice for the evaluation \& treatment of male sexual dysfunction, a couple's problem. Endocrinol pract 2003; 9(1): 7895

22. Husain A, Virmani OP, Popli SP, Misra LN, Gupta MM, Srivastava GN et al. Dictionary of Indian Medicinal Plants, IMAP. Lucknow, India, 1992,546

23. Jain DL, Baheti AM, Jain RS, Khandelwal RK. Use of medicinal plants among tribes in Satpuda region of Dhule and Jalgaon districts of Maharashtra-An Ethanobotanical survey. Indian Journal of Traditional Knowledge 2010; 9(1):152-157.

24. Jaiswal S, Singh SV, Singh B, Singh HN. Plants used for tissue healing of animals. Natural Products Radiance 2004; 3(4):284-292.

25. Joy PP, Thomas J, Mathew S, Skaria PB. Medicinal Plants, Kerala Agricultural University. Aromatic and Medicinal Plants Research Station, 1998

26. Kapoor LD, CRC Handbook of Med. Ayurvedic Plants. CRC Press, 1990, 18.

27. Karntchouing P, Mbongue GYF, Dimo T, Watcho P, Jatsa HB, Sokeng SD (2002). Effects of Aframomum melegueta \& Piper guineense on sexual behavior of male rats. Behav. Pharmacol. 13: 243-247.

28. Khan \& Khan. Folk medicines for male sexual disorders. Indian J Traditional Knowledge 2005; 4(3):317-324

29. Klinger T, Noe S, Riischke J, Muller S, O Benkeir. Effects of of Yohimbine on sexual experiences \& nocturnal penile tumescence \& rigidity in erectile dysfunction. Arch Sex Behav. 1996; 16: 111-124.

30. McKinlay JB, The worldwide prevalence of epidemiology. International Journal of Impotence Research, 2000: 12(S4) S6.

31. Maiti S, Geetha KA. Horticulture Floriculture (Ornamental, Medicinal \& Aromatic Crops) Medicinal and Aromatic Plants in India, 2007

32. Malviya N, Jain S, Gupta VB, Vyas S. Indigenous Herbal remedies used by tribals of Madhya Pradesh for Improving their Sexual Performance and Problem associated with Sexuality. IJRAP. 2011, 2(2):399-402.

33. Malviya N., S. Jain, VB Gupta, S Vyas : Acta Poloniae Pharmaceutica Drug Research, Vol. 68 No. 1 pp. 3-8, 2011

34. Meena KA, Bansal P, Kumar S. Plants-herbal wealth as a potential source of ayurvedic drugs. Asian Journal of Traditional Medicines 2009:4(4):152-170.

35. Mugisha MK, Origa HO. Traditional herbal remedies used in the management of sexual impotence and erectile dysfunction in western Uganda. African Health Sciences 2005; 5(1):40-49

36. Neychev VK, Mitev VI. The aphrodisiac herb Tribulus terrestris does not influence the androgen production in young men. J Ethnopharmacol 2005; 101:319-323. 
Indo Global Journal of Pharmaceutical Sciences, 2019; 9(2): 91-97

37. Neychev VK, Mitevvl, 2000. The aphrodisiac herb. Tribulus terrestris does not influence the androgen production in young men. J ethnopharmacal, 2005: 101: 319-23

38. Orisakwe OE, Husaini DC, Afonne OJ (2004). Testicular effects of subchronic administration of Hibiscus sabdariffa calyx aqueous extract in rats. Reprod. Toxicol. 18(2): 295-298

39. Pallavi K J, Ramadeep S, Sarbjeet S, Kavam S, Mamta F, Vinod S. J Aphrodisiac agents from Medicinal Plants: A review, J. Chem Pharm. Res, 2016; 3: 911-21

40. Patel D.K, R Kumar, SK Prasad, K Sairam, S Hemalatha., Asian Pacific Journal of Tropical Biomedicine, S131-S138, 2011

41. Prasanth PR, Kumar A. Ethno-medico botany of medicinal plants for the treatment of diabetic activity in Krishna district, Andhra Pradesh. International Journal of Pharma Research and Development - Online 2008; 9:1-9

42. Singh R., Singh S, Jeyabalan G, Ashraf Ali. An overview of Traditional Medicinal Plants as Aphrodisiac Agent. J. of Pharmacognosy and Phytochemistry. 2012: Vol. 1, issue 4, 43-56.

43. Ratrasooriya WD, Dharmasiri MG. Effect of Terminalia cattapa seeds on sexual behavior and fertility of male Rats. Asian Journal of Andrology: 2000, 213-219

44. Sankaranarayanan S, Bama P, Ramachandran J, Kalaichelvan TP, Deccaraman M, Vijayalakshimi $M$ et al. Ethnobotanical study of medicinal plants used by traditional users in Villupuram district of Tamil Nadu, India. Journal of Medicinal Plants Research 2010; 4(12):1089-1101.

45. Satyavati GV, Raina MK, Sharma M. Medicinal Plants of India. Ind Council on Med Res 1976; 1

46. Sharma BD, S. Karthideyan and NP Singh. Flora of Maharashtra state, Monocotyledones. Volume 2: 1996

47. Sharma VN. Some observations on hypoglycaemic activity of Momordica charantia. Indian J Med Res 1960; 48(4):471-47.

48. Singh KP, Kumar V, Tiwari KR, Sharma A, Rao CV, Singh RH. Medico-Ethnobotany of 'Chatara' Block of District Sonebhadra, Uttar Pradesh, India. Advances in Biological Research 2010; 4(1):65-80.

49. Singh KP, Singh PA, Gupta KA, Chaudhary S. Beneficial effects of aqueous fruit extract of Tribulus terrestris on testicular and serum biochemistry of albino rats. J Ecophysiol Occup Hlth 2009; 9:217-223.

50. Singh NP, P. Lakshminarasimhan, S. Karthikeyan and PV Prasana. Flora of Maharashtra state, Dicotyledones. Volume 2: 2001

51. Singh NP and S. Karthikeyan. Flora of Maharashtra state, Dicotyledones. Volume 1: 2000

52. Subramoniam A, Madhavachandran V, Ravu K, Anuja VS. Aphrodisiac property of elephant creeper Argyreia nervosa. Journal of Endocrinology \& Reproduction 2007; 11(2):82-85

53. Sumalatha K, Kumar SA, Lakshmi SM. Review on Natural Aphrodisiac potentials to treat Sexual dysfunction. International Journal of Pharmacy \&Therapeutics 2010; 1:10-18.

54. Parial S., Jain DC, Joshi SB. Antidiabetic activity of Ficus retusa leaves Drug Invention today. 2010, (1):96-101

55. Thomas J, Joy PP, Mathew S, Skaria BP, Duethi PP, Joseph TS Agronomic Practices for Aromatic and Medicinal Plants Kerala Agricultural University and Directorate of Arecanut \& Spices Development (Min. of Agri., Govt. of India). Calicut, Kerala, India, 2000

56. Vanwyk BE, Gericke N. People's plants: a guide to useful plants of southern Africa, Briza Publications, Pretoria, South Africa, 2000, 351

57. Willaman JJ, Li HL. Alkaloid bearing plants and their contained alkaloids. J Nat Prod 1970; Suppl 33(3A).

58. Woys WW. Heirloom Vegetable Gardening. Henry Holt and Company, New York, 1997

59. Yakubu MT, Akanji MA, Oladiji A. Male sexual dysfunction \& methods used in assessing medicinal plants with aphrodisiac potentials. Phcog. Rev. 2007; 11: 49-56

60. Yakubu MT, Akanji MA, Oladiji A. Male sexual dysfunction \& methods used in assessing medicinal plants with aphrodisiac potentials. Phcog. Rev. 2007; 11: 49-56.

61. Yakubu MT, Bilbis LS, Lawal M, Akanji MA. Effect of repeated administration of Sildenafil citrate on selected enzyme activities of liver $\&$ kidney of male albino rats. Nigji pure \& appl Sci 2003; 18: 1395 4000

62. Yakubu MT, Bilbis LS, Lawal M, Akanji MA. Effect of repeated administration of Sildenafil citrate on selected enzyme activities of liver \& kidney of male albino rats. Nigji pure \& appl Sci 2003; 18: 1395 4000 .

Indo Global Journal of Pharmaceutical Sciences( ISSN 2249 1023; CODEN- IGJPAI; NLM ID: 101610675) indexed and abstracted in CrossRef (DOI Enabling), UGC CARE Journal List, EMBASE(Elsevier), National Library of Medicine (NLM) Catalog, ResearchGate, Publons, CAS (ACS), Index Copernicus, Google Scholar and many more. For further details, visit http://iglobaljournal.com

This is a special issue as an outcome of 'International Conference on Recent Advances in Traditional Medicine, Medicinal Plants and Phytochemistry' jointly organized by Ahmednagar College, India and AIMST University, Malaysia. Relaxation offered in journal format. 